\title{
Investigation of the Predictive Values of Triglyceride/HDL Cholesterol Ratio and Whole Blood Viscosity with Regard to Severe Peripheral or Carotid Artery Disease in Patients Scheduled for Coronary Bypass
}

\author{
Mesut Engin, $M D,{ }^{1}$ Orhan Güvenç, $\mathrm{MD}^{2}$ \\ ${ }^{1}$ Department of Cardiovascular Surgery, University of Health Sciences, Mehmet Akif İnan Training and Research Hospital, Şanlıurfa, \\ Turkey; ${ }^{2}$ Department of Cardiovascular Surgery, Medical Faculty of Uludağ University, Bursa, Turkey
}

\section{ABSTRACT}

Background: The incidence of atherosclerotic cardiovascular diseases, which constitute an important disease group, is expected to rise with increasing life expectancy. The aim of this study was to investigate the predictive values of triglyceride/HDL cholesterol (TG/HDL-C) ratio and whole blood viscosity on possible severe carotid or peripheral arterial disease in patients who were admitted to our clinic and scheduled for coronary artery bypass graft (CABG) surgery.

Methods: This study retrospectively examined 299 patients who were admitted to our clinic and scheduled for CABG between August 1, 2015, and August 1, 2019. Patients with severe carotid or peripheral arterial disease were included in group 2; those in whom peripheral arterial disease and stenosis were not detected were included in group 1.

Results: Group 1 consisted of 255 patients, mean ( \pm standard deviation) age $58.1 \pm 8.9$ years; the 44 patients in group 2 had a mean age of $64.1 \pm 10.6$ years $(P=.018)$. The incidence of diabetes mellitus and tobacco use were significantly higher in group $2(P<.001$ and $P=.034$, respectively). Triglyceride values, TG/HDL-C, and whole blood viscosity at high and low shear rates were significantly higher in group $2(P=.017, P<.001, P<.001$, and $P<.001$, respectively). Receiver operator characteristic analysis revealed that the cutoff value of TG/HDL-C was 3.2 with $77 \%$ sensitivity and $72 \%$ specificity; that of high-shear-rate viscosity was 16.1 with $73 \%$ sensitivity and $69 \%$ specificity; and that of low-shear-rate viscosity was 59.2 with $70 \%$ sensitivity and $63 \%$ specificity.

Conclusion: It is possible to predict severe additional arterial diseases in patients scheduled for CABG with whole blood viscosity and TG/HDL-C ratios, both of which can be obtained with simple blood tests.

Received April 3, 2020; accepted April 21, 2020.

Correspondence: Mesut Engin, MD, Department of Cardiovascular Surgery, University of Health Sciences, Mehmet Akif Inan Training and Research Hospital, Esentepe Mah, Ertuğrul Cad, 63200, Karaköprü/Şanlurfa, Türkiye (e-mail:mesut_kvc_cor@hotmail.com).

\section{INTRODUCTION}

The incidence of atherosclerotic cardiovascular diseases, which constitute an important disease group, is expected to rise with increasing life expectancy. Among these, coronary artery disease is the most commonly seen. Despite widespread usage of endovascular methods, coronary artery bypass graft (CABG) surgery is the gold standard treatment method in various cases of vascular and main coronary diseases [Hillis 2011; Teker Açikel 2019]. A thorough examination and preparation of patients is essential before CABG. The preoperative diagnosis of possible comorbidities, such as severe carotid or peripheral artery disease, which may affect surgical strategy, is of foremost importance. For example, the team may preoperatively determine the possible intra-aortic balloon pump (IABP) placement method in a patient with low ejection fraction and bilaterally occluded iliac arteries. Also, a case with severe carotid artery stenosis (CAS) may necessitate a revision in surgical strategy.

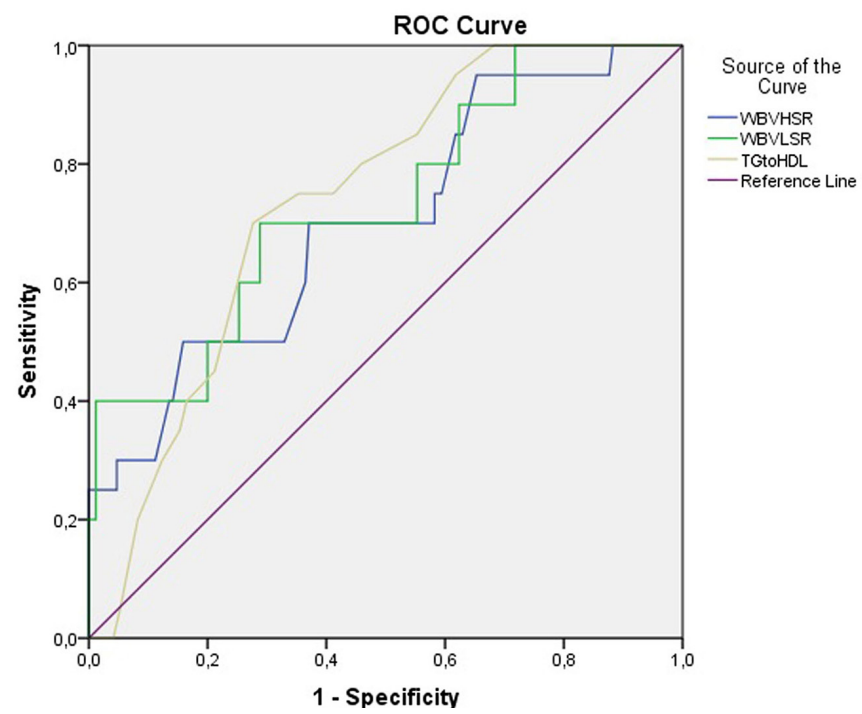

ROC curve and AUC for TG/HDL-C, WBVLSR, and WBVHSR for predicting concomitant severe carotid artery disease or PAD in patients scheduled to undergo CABG. TG/HDL-C cutoff 3.2, 77\% sensitivity, $72 \%$ specificity; WBVHSR cutoff 16.1 , 73\% sensitivity, $69 \%$ specificity; WBVLSR cutoff 59.2 , 70\% sensitivity, $63 \%$ specificity. 
Table 1. Demographic Data of the Patients*

\begin{tabular}{lccc}
\hline Variable & $\begin{array}{c}\text { Group 1 } \\
(\mathrm{n}=255)\end{array}$ & $\begin{array}{c}\text { Group } 2 \\
(\mathrm{n}=44)\end{array}$ & $P$ \\
\hline Age $(\mathrm{y})$ & $58.1 \pm 8.9$ & $64.1 \pm 10.6$ & $.018 \dagger$ \\
Ejection fraction $(\%)$ & $50.4 \pm 6.9$ & $48.6 \pm 7.1$ & .214 \\
Body mass index $\left(\mathrm{kg} / \mathrm{m}^{2}\right)$ & $29.3 \pm 3.9$ & $28.9 \pm 4.1$ & .356 \\
Male sex & $162(63.5)$ & $32(72.7)$ & .686 \\
Previous PCI & $82(32.1)$ & $18(40.9)$ & .499 \\
COPD & $55(21.5)$ & $11(25)$ & .866 \\
Hypertension & $163(63.9)$ & $27(61.3)$ & .827 \\
Diabetes mellitus & $71(27.8)$ & $29(65)$ & $<.001 \dagger$ \\
Tobacco use & $90(35.3)$ & $26(59)$ & $.034 \dagger$ \\
ASA use & $87(34.1)$ & $19(43.1)$ & .481 \\
ACEl/ARB use & $76(29.8)$ & $14(31.8)$ & .969 \\
Statin use & $69(27)$ & $10(23)$ & .480 \\
Beta-blocker use & $66(25.8)$ & $12(27.2)$ & .623 \\
\hline
\end{tabular}

*Data are mean $\pm \mathrm{SD}$ or $\mathrm{n}(\%)$. ACEl indicates angiotensin-converting enzyme inhibitor; ARB, angiotensin receptor blocker; ASA, acetylsalicylic acid; COPD, chronic obstructive pulmonary disease; $\mathrm{PCl}$, percutaneous coronary intervention.

†Statistically significant at $P<.05$.

Easily obtainable from routine blood tests of the patients, the ratio of triglycerides to high-density-lipoprotein cholesterol (TG/HDL-C) ratio, also known as the atherogenic index, is reportedly related to mortality in cardiovascular diseases [Chang 2017; Frohlich 2003]. In addition, it has been shown to correlate with disease severity in patients who underwent coronary angiography. Whole blood viscosity (WBV) is the main determinant factor of endothelial shear stress (ESS) and is considered a predictor of many cardiovascular diseases, such as hypertension and hyperlipidemia [Cecchi 2011; Ozcan Cetin 2015].

The aim of this study was to investigate the predictive values of the TG/HDL-C ratio and whole blood viscosity on possible severe carotid or peripheral arterial disease in patients who were admitted to our clinic and scheduled for CABG.

\section{METHODS}

This study retrospectively examined 299 patients who were admitted to Mehmet Akif İnan Training and Research Hospital, Department of Cardiovascular Surgery, and scheduled for CABG between August 1, 2015, and August 1,2019. The study was approved by the institutional review board. The patients were divided into 2 groups after preoperative evaluations: group 1 consisted of patients with coronary artery disease only, and group 2 included those with stage C/D (according to Trans Atlantic
Inter-Society Consensus-II [TASC-II] classification) aortoiliac or femoropopliteal diseases or severe carotid artery diseases. Emergency operations, reoperations, patients who required additional cardiac surgery, patients with chronic renal failure or any known systemic inflammatory or rheumatic diseases were excluded from the study. Patient data were gathered from the hospital registry and patient files. All patients' demographic characteristics, hemograms, biochemical parameters, and preoperative examinations regarding peripheral artery or carotid artery systems were recorded.

\section{Measurement of Blood Parameters}

The blood samples of all patients who admitted to our clinic with planned surgeries were obtained from peripheral veins, and EDTA-coated test tubes were used for analysis. Automatic and molecular analyzers were used for the measurement of hematologic and biochemical parameters, respectively. TG/HDL-C ratio and whole blood viscosity were calculated from the results of these analyses.

\section{Calculation of Whole Blood Viscosity}

Two values of whole blood viscosity were calculated at high shear rate (WBVHSR) and at low shear rate (WBVLSR). Total protein (TP) and hematocrit (HCT) parameters were used for the following formulas [Nwose 2010]:

WBVHSR $\left(208 \mathrm{~s}^{-1}\right)=(0.12 \mathrm{HCT}[\%])+0.17(\mathrm{TP}[\mathrm{g} / \mathrm{L}]-2.07)$; $\operatorname{WBVLSR}\left(0.5 \mathrm{~s}^{-1}\right)=(1.89 \mathrm{HCT}[\%])+3.76(\mathrm{TP}[\mathrm{g} / \mathrm{L}]-78.42)$.

\section{Diagnosis of Severe Carotid or Peripheral Artery Disease}

During preoperative preparation, all patients underwent carotid artery Doppler ultrasonography (DUSG). All patients with internal carotid artery peak systolic flow velocity (PSV) $>125 \mathrm{~cm} / \mathrm{s}$ (>50\% stenosis) or ICA PSV/common carotid artery PSV >2 were evaluated by computed tomographic angiography or conventional angiography. Carotid artery stenosis levels were determined according to the North American Symptomatic Carotid Endarterectomy ratings, and accordingly, 1 -sided $\geq 70 \%$ stenosis was considered serious carotid artery disease (22 patients). Before the operation, detailed examinations of the patients whose coronary bypass was planned for the diagnosis of peripheral artery disease were performed. Doppler ultrasonography was performed in all patients with pathological findings in peripheral pulses and in those with ankle brachial index $<0.9$. Patients with $\geq 50 \%$ stenosis findings in the peripheral arteries were evaluated by computed tomographic angiography or conventional angiography. Based on this examination, TASC classification [Norgren 2007] was made, and those with stage C/D disease were evaluated as patients with serious peripheral artery disease (14 patients). Eight patients had both severe carotid and peripheral arterial diseases.

\section{Statistical Analysis}

SPSS 21.0 (IBM, version 21.0, Chicago, IL) was used for data analysis. Continuous and ordinal data were presented as mean and standard deviation. Kolmogorov-Smirnov and Shapiro-Wilk tests were used the evaluation of normality of 
Table 2. Laboratory Variables of the Patients*

\begin{tabular}{|c|c|c|c|}
\hline Variables & $\begin{array}{l}\text { Group } 1 \\
(n=255)\end{array}$ & $\begin{array}{l}\text { Group } 2 \\
(n=44)\end{array}$ & $P$ \\
\hline White blood cells $(103 / \mu \mathrm{L})$ & $9.7 \pm 3.9$ & $10.9 \pm 4.1$ & .131 \\
\hline Hematocrit (\%) & $40.1 \pm 4.9$ & $39.3 \pm 5.2$ & .414 \\
\hline Platelets $\left(10^{3} / \mu \mathrm{L}\right)$ & $270.3 \pm 71.4$ & $291.7 \pm 62.8$ & .216 \\
\hline Neutrophils $\left(10^{3} / \mu \mathrm{L}\right)$ & $6.2 \pm 1.3$ & $6.6 \pm 1.2$ & .615 \\
\hline Lymphocytes $\left(10^{3} / \mu \mathrm{L}\right)$ & $2.7 \pm 1.3$ & $2.3 \pm 1.1$ & .314 \\
\hline Total protein $(\mathrm{g} / \mathrm{dL})$ & $6.9 \pm 0.4$ & $7.2 \pm 0.3$ & .060 \\
\hline Albumin (mg/dL) & $4.2 \pm 0.3$ & $4.3 \pm 0.4$ & .393 \\
\hline Urea $(\mathrm{mg} / \mathrm{dL})$ & $31.4 \pm 8.1$ & $33.1 \pm 7.9$ & .224 \\
\hline Creatinine $(\mathrm{mg} / \mathrm{dL})$ & $0.97 \pm 0.23$ & $0.99 \pm 0.3$ & .182 \\
\hline C-reactive protein $(\mathrm{mg} / \mathrm{dL})$ & $8.8 \pm 9.7$ & $9.1 \pm 10.7$ & .096 \\
\hline Total cholesterol (mg/dL) & $189.4 \pm 66.9$ & $195.8 \pm 57.8$ & .260 \\
\hline LDL cholesterol (mg/dL) & $124.7 \pm 41.9$ & $126.9 \pm 37.6$ & .456 \\
\hline HDL cholesterol (mg/dL) & $42.2 \pm 7.3$ & $39.8 \pm 8.7$ & .074 \\
\hline Triglycerides (mg/dL) & $181.9 \pm 86.7$ & $193.9 \pm 74.7$ & $.017 \dagger$ \\
\hline TG/HDL-C & $2.5 \pm 0.9$ & $3.7 \pm 0.5$ & $<.001 \dagger$ \\
\hline WBVHSR & $15.46 \pm 1.50$ & $16.84 \pm 1.62$ & $<.001 \dagger$ \\
\hline WBVLSR & $51.96 \pm 16.08$ & $66.29 \pm 17.41$ & $<.001 \dagger$ \\
\hline
\end{tabular}

*Data are mean \pm SD.

†Statistically significant at $P<.05$.

distribution. Student's t test and Mann-Whitney U test were used for normally and non-normally distributed data, respectively. Frequency and percentage analysis were performed for nominal variables, which were compared with $\chi^{2}$ test. Multivariate binary logistic regression analysis was used to analyze the predictors of peripheral and carotid artery stenosis. Receiver operating characteristic (ROC) curve analysis was performed to evaluate the predictive values of whole blood viscosity and TG/HDL-C ratio in comorbid additional severe arterial diseases, and the area under the curve (AUC) was calculated.

\section{RESULTS}

The demographic data of 299 patients are presented in Table 1. Group 1 consisted of 255 patients, mean age 58.1 \pm 8.9 years. The 44 patients in group 2 had a mean age of $64.1 \pm 10.6$ years $(P=.018)$. The 2 groups were similar in terms of sex, body mass index, hypertension, chronic obstructive pulmonary disease (COPD), and current medication (eg, statin and beta blocker use). The incidence of diabetes mellitus (DM) and tobacco use were significantly higher in group $2(P<.001$ and $P=.034$, respectively).

The laboratory parameter data of the patients are presented in Table 2. There was no significant difference between
Table 3. Multivariate Logistic Regression Analysis to Predict Severe Peripheral or Carotid Arterial Disease in Patients Scheduled to Undergo CABG

\begin{tabular}{llll}
\hline Variable & Adjusted OR & $95 \% \mathrm{Cl}$ & $P$ \\
\hline Model 1 & & & \\
Age & 1.085 & 1.014 to 1.160 & $.038 \dagger$ \\
Triglycerides & 1.017 & 0.999 to 1.034 & .065 \\
Tobacco use & 0.455 & 0.156 to 1.021 & .114 \\
DM & 2.651 & 1.006 to 2.998 & $.009 \dagger$ \\
TG/HDL-C & 3.426 & 1.316 to 8.921 & $.012 \dagger$ \\
WBVHSR & 2.161 & 1.382 to 3.378 & $.001 \dagger$ \\
Model 2 & & & \\
Age & 1.070 & 1.010 to 1.158 & $.047 \dagger$ \\
Triglycerides & 1.013 & 0.996 to 1.030 & .128 \\
DM & 2.415 & 1.001 to 2.644 & $.011 \dagger$ \\
Tobacco use & 0.354 & 0.120 to 1.047 & .061 \\
TG/HDL-C & 3.886 & 1.458 to 10.353 & $.007 \dagger$ \\
WBVLSR & 1.063 & 1.023 to 1.105 & $.002 \dagger$ \\
\hline
\end{tabular}

†Statistically significant at $P<.05$.

the 2 groups in terms of hemogram parameters, total protein, albumin, urea, creatinine, C-reactive protein, total cholesterol, HDL cholesterol, and LDL cholesterol (LDL-C). Triglyceride values, TG/HDL-C, WBVHSR, and WBVLSR were significantly higher in group $2(P=.017, P<.001$, $P<.001$, and $P<.001$, respectively).

In patients scheduled for $\mathrm{CABG}$, multivariate logistic regression analysis was performed in 2 different models with respect to WBVHSR and WBVLSR values to evaluate additional severe PAD or CAS (Table 3). Age, DM, TG/HDL-C ratio (odds ratio [OR] 3.426, 95\% confidence interval [CI] 1.316 to $8.921, P=.012$ ) and WBVHSR (OR 2.161, 95\% CI 1.382 to $3.378, P=.001)$ were identified as the independent predictors of cormorbid serious arterial disease in model 1 . Age, DM, TG/HDL-C ratio (OR 3.386, 95\% CI 1.458 to $10.353, P=.007)$ and WBVLSR (OR $1.063,95 \%$ CI 1.023 to $1.105, P=.002)$ were the independent predictors in model 2 .

ROC curve analysis revealed that the cutoff value of TG/ HDL-C was 3.2 with $77 \%$ sensitivity and $72 \%$ specificity; that of WBVHSR was 16.1 with $73 \%$ sensitivity and $69 \%$ specificity; and that of WBVLSR was 59.2 with $70 \%$ sensitivity and $63 \%$ specificity (Figure).

\section{DISCUSSION}

Coronary artery bypass operations are major surgeries. One of the principal factors affecting the success of the operation is the preoperative preparation of the patients, which includes their systematic evaluation. Physicians must beware 
of carotid and peripheral artery disease in patients scheduled for CABG, because they may cause serious morbidity and mortality. With our current study, we detected, for the first time in the literature, that WBV and TH/HDL-C ratio, which are easily obtainable from routine blood parameters, may predict serious additional arterial diseases in patients scheduled for CABG.

Atherosclerosis is the basis of cardiovascular diseases, and various inflammatory mechanisms play a role in its pathophysiology [Xing 2019]. Epidemiological research has shown that increased blood lipid values are a risk factor for atherosclerotic heart diseases [Di Angelantonio 2009; Sarwar 2007]. Further studies report that TG/HDL-C ratio has a higher predictive value than a single blood lipid parameter [Asia Pacific Cohort Studies Collaboration 2005]. HDL cholesterol has protective effects against atherosclerosis, the most commonly known being the transportation of cholesterol from macrophages on vessel walls to the liver [Cuchel 2006]. Additionally, it induces vasorelaxation and contributes to protecting the hemostasis of endothelial cells by inhibiting cellular adhesion, thereby preventing the migration of proinflammatory cytokines to vascular endothelia [Calabresi 2003]. In contrast, increased TG values are a risk factor for atherosclerosis. Puri et al [2016] detected a direct correlation between increased TG values and atherosclerosis. In light of this data, it is safe to predict that a rise in TG/HDL-C ratio poses an important risk factor for atherosclerosis.

In the prospective cohort study of Chen et al [2020] on 96,542 volunteers, the association of TG/HDL-C with cardiovascular events was investigated over a 9-year follow-up period, during which 5422 cardiovascular events occurred in patients whose TG/HDL-C ratios were significantly increased. Chi et al [2018] conducted a study in 1599 individuals $>65$ years of age, examining the efficiency of lipid parameters in reflecting target organ damage, specified as progression in peripheral arterial disease, aortic thickening, or renal damage occurring due to atherosclerosis. The results of the study showed that TG/HDL-C and non-HDL-C values were more effective in reflecting target organ damage compared with other lipid parameters [Chi 2018]. In light of these data, we determined that increased TG/HDL ratio could predict severe comorbid carotid and peripheral artery disease in patients scheduled for CABG in our clinic and calculated the cutoff value as 3.2 with $77 \%$ sensitivity and $72 \%$ specificity based on ROC curve analysis.

The pathophysiology of atherosclerosis is complex and is yet to be understood. Hyperlipidemia, DM, and smoking are known risk factors. However, half of the patients with myocardial infarct do not have hyperlipidemia, and traditional risk factors cannot be identified in $20 \%$. Therefore, other mechanisms are suspected to underlie this condition, which is how whole blood viscosity came to be researched. Whole blood viscosity is a marker of ESS and is closely related to cardiovascular diseases. Hyperviscosity is pathophysiologically similar to the traditional risk factors such as hypertension, smoking, DM, male sex, and hyperlipidemia [Sloop 2015].

As one of the important determinants of ESS, WBV plays a critical role in maintaining normal endothelial function. Increased ESS may disrupt microvascular circulation owing to thrombogenic events that occur with atherosclerotic plaque rupture and may cause major events in cerebral, cardiac, and peripheral circulation [Cecchi 2011; Kim 2013]. In the normally functioning vascular endothelia, mild increases in viscosity induce the release of nitric oxide (NO), thereby ensuring circulation continuity. However, in cases of endothelial dysfunction due to atherosclerotic changes, $\mathrm{NO}$ release is disrupted, resulting in circulatory disorders resulting from increased viscosity [Salazar Vázquez 2010]. Infiltration of lipid particles and fibrinogen to vascular endothelia by the cause of increased WBV is another mechanism leading to this pathophysiology [Marossy 2009].

In the Edinburgh Artery study, along with risk factors such as hypertension and hyperlipidemia, WBV was found to correlate with cardiovascular events and stroke [Lowe 1997]. In patients who developed ST-elevated myocardial infarct, Cetin et al [2016a] investigated the prognostic importance of WBV during a mean follow-up time of 34.6 months and found that increased WBV values were related to shortand long-term major adverse events. The West of Scotland Coronary Prevention Study detected a direct correlation between increased WBV and coronary artery risk factors as well as peripheral and coronary atherosclerosis [Lowe 2000]. In another study, the relationship between WBV and coronary collateral circulation was investigated. Coronary artery disease patients with total occlusion were divided into 2 groups based on collateral circulation, and the risk factors for insufficient collateral circulation were researched. Multivariate logistic analysis revealed that both WBVHSR and WBVLSR were independent predictors for insufficient development of collateral circulation. The cutoff values for WBVLSR and WBVHSR were 67.1 (sensitivity $73 \%$, specificity $67.1 \%$ ) and 16.8 (sensitivity $70 \%$, specificity $70.5 \%$ ), respectively [Cetin 2016b]. Similarly, we detected a correlation between WBV and severe additional arterial disease in our group of coronary artery disease patients.

Possible severe CAS may increase the cerebrovascular event risk. Even if no intervention is planned for the carotid artery, being aware of it preoperatively may allow the team to take precautions such as maintaining a high perfusion pressure during surgery. Peripheral artery disease may also pose risks during $\mathrm{CABG}$ operations and should be diagnosed preoperatively. Ignorance of possible severe aortoiliac diseases may cause difficulty in the placement of mechanical support systems (IABP, etc) after the operation. In addition, distal perfusion may be impaired during coronary bypass in patients with severe femoropopliteal lesions [Te Kolste 2015]. We showed that WBV and TG/HDL-C ratio, both of which can be obtained with simple blood tests, may predict possible severe additional arterial diseases in patients scheduled for CABG.

The retrospective nature of this study is its most prominent limitation. In addition, although routine carotid DUSG is preoperatively performed to all patients in our clinic, peripheral artery DUSG is not a routine practice. Also, because physical examination is subjective, the diagnosis of PAD may have been omitted in a small number of patients. 
In conclusion, it is possible to predict severe additional arterial diseases in patients scheduled for CABG with WBV and TG/HDL-C ratio, both of which can be obtained with simple blood tests.

\section{REFERENCES}

Asia Pacific Cohort Studies Collaboration. 2005. A comparison of lipid variables as predictors of cardiovascular disease in the Asia Pacific Region. Ann Epidemiol 15:405-413.

Calabresi L, Gomaraschi M, Franceschini G. 2003. Endothelial protection by high-density lipoproteins: From bench to bedside. Arterioscler Thromb Vasc Biol 23:1724-1731.

Cecchi E, Giglioli C, Valente S, et al. 2011. Role of hemodynamic shear stress in cardiovascular disease. Atherosclerosis 214:249-256

Cetin EH, Cetin MS, Canpolat U, et al. 2016a. Prognostic significance of whole blood viscosity estimated by de Simone's formula in ST-elevation myocardial infarction. Biomark Med 10:495-511.

Cetin MS, Çetin EHÖ, Balcı KG, et al. 2016b. The association between whole blood viscosity and coronary collateral circulation in patients with chronic total occlusion. Korean Circ J 46:784-790.

Chang TI, Streja E, Soohoo M, et al. 2017. Association of serum triglyceride to HDL cholesterol ratio with all-cause and cardiovascular mortality in incident hemodialysis patients. Clin J Am Soc Nephrol 12:591-602.

Chen Z, Chen G, Qin H, et al. 2020. Higher triglyceride to high-density lipoprotein cholesterol ratio increases cardiovascular risk: 10-year prospective study in a cohort of Chinese adults. J Diabetes Investig $11: 475-481$

Chi C, Teliewubai J, Lu YY, et al. 2018. Comparison of various lipid parameters in association of target organ damage: A cohort study. Lipids Health Dis 17:199.

Cuchel M, Rader DJ. 2006. Macrophage reverse cholesterol transport: Key to the regression of atherosclerosis? Circulation 113:2548-2555.

Di Angelantonio E, Sarwar N, Perry P, et al. 2009. Emerging Risk Factors Collaboration. Major lipids, apolipoproteins, and risk of vascular disease. JAMA 302:1993-2000.

Frohlich J and Dobiasova M. 2003. Fractional esterifcation rate of cholesterol and ratio of triglycerides to HDL-cholesterol are powerful predictors of positive findings on coronary angiography. Clin Chem 49:1873-1880.

Hillis LD, Smith PK, Anderson JL, et al. 2011. 2011 ACCF/AHA Guideline for Coronary Artery Bypass Graft Surgery: A report of the American College of Cardiology Foundation/American Heart Association Task
Force on Practice Guidelines. Circulation 124:e652-e735.

Kim J, Chung H, Cho M, et al. 2013. The role of critical shear stress on acute coronary syndrome. Clin Hemorheol Microcirc 55:101-109.

Lowe GD, Lee AJ, Rumley A, et al. 1997. Blood viscosity and risk of cardiovascular events: The Edinburgh Artery Study. Br J Haematol 96:168-173.

Lowe G, Rumley A, Norrie J, et al. 2000. Blood rheology, cardiovascular risk factors, and cardiovascular disease: The West of Scotland Coronary Prevention Study. Thromb Haemost 84:553-558.

Marossy A, Svorc P, Kron I, Gresová S. 2009. Hemorheology and circulation. Clin Hemorheol Microcirc 42:239-258.

Norgren L, Hiatt WR, Dormandy JA, et al. 2007. TASC II Working Group. Inter-Society Consensus for the Management of Peripheral Arterial Disease (TASC II). J Vasc Surg 45(suppl S):S5-S67.

Nwose EU. 2010. Cardiovascular risk assessment and support techniques: Whole blood viscosity assessment issues I: extrapolation chart and reference values. North Am J Med Sci 2:165-169.

Ozcan Cetin EH, Cetin MS, Canpolat U et al. 2015. The forgotten variable of shear stress in mitral annular calcifcation: Whole blood viscosity. Med Princ Pract 24:444-450.

Puri R, Nissen SE, Shao M, et al. 2016. Non-HDL cholesterol and triglycerides: Implications for coronary atheroma progression and clinical events. Arterioscler Thromb Vasc Biol 36:2220-2228.

Salazar Vázquez BY, Martini J, Chávez Negrete A, et al. 2010. Cardiovascular benefits in moderate increases of blood and plasma viscosity surpass those associated with lowering viscosity: Experimental and clinical evidence. Clin Hemorheol Microcirc 44:75-85.

Sarwar N, Danesh J, Eiriksdottir G, et al. 2007. Triglycerides and the risk of coronary heart disease: 10,158 incident cases among 262,525 participants in 29 Western prospective studies. Circulation 115:450-458.

Sloop G, Holsworth RE Jr, Weidman JJ, St Cyr JA. 2015. The role of chronic hyperviscosity in vascular disease. Ther Adv Cardiovasc Dis 9:19-25.

Te Kolste HJ, Balm R, de Mol B. 2015. Acute compartment syndrome of the lower leg after coronary artery bypass grafting: A silent but dangerous complication. Thorac Cardiovasc Surg 63:300-306.

Teker Açıkel ME, Korkut AK. 2019. Impact of Controlling Nutritional Status Score (CONUT) and Prognostic Nutritional Index (PIN) on patients undergoing coronary artery bypass graft surgery. Heart Surg Forum 22:E294-E297.

Xing Y, Guo JT, Gai LY, et al. 2019. Association of uric acid and C-reactive protein with the severity of coronary artery disease using SYNTAX Score and Clinical SYNTAX Score. Heart Surg Forum 22:E247-E252. 